\title{
Foundations of Hardware-Based Attested Computation and Application to SGX
}

\author{
Manuel Barbosa *, Bernardo Portela ${ }^{\dagger}$, Guillaume Scerri ${ }^{\ddagger}$, Bogdan Warinschi ${ }^{\S}$ \\ * HASLab, INESC TEC, FCUP \\ Email: mbb@dcc.fc.up.pt \\ $\dagger$ HASLab, INESC TEC, UMinho \\ Email: blfportela@gmail.com \\ $\ddagger$ University of Bristol \\ Email: guillaume.scerri@bris.ac.uk \\ $\S$ University of Bristol \\ Email: csxbw@bristol.ac.uk
}

\begin{abstract}
Exciting new capabilities of modern trusted hardware technologies allow for the execution of arbitrary code within environments completely isolated from the rest of the system and provide cryptographic mechanisms for securely reporting on these executions to remote parties.

Rigorously proving security of protocols that rely on this type of hardware faces two obstacles. The first is to develop models appropriate for the induced trust assumptions (e.g., what is the correct notion of a party when the peer one wishes to communicate with is a specific instance of an an outsourced program). The second is to develop scalable analysis methods, as the inherent stateful nature of the platforms precludes the application of existing modular analysis techniques that require high degrees of independence between the components.

We give the first steps in this direction by studying three cryptographic tools which have been commonly associated with this new generation of trusted hardware solutions. Specifically, we provide formal security definitions, generic constructions and security analysis for attested computation, key-exchange for attestation and secure outsourced computation. Our approach is incremental: each of the concepts relies on the previous ones according to an approach that is quasi-modular. For example we show how to build a secure outsourced computation scheme from an arbitrary attestation protocol combined together with a key-exchange and an encryption scheme.
\end{abstract}

\section{Introduction}

BACKGROUND. The many applications that routinely manipulate sensitive data require strong guarantees which ensure i. that adversaries cannot tamper with their execution; and ii. that no sensitive information is leaked. Yet, satisfying these guarantees on modern execution platforms rife with vulnerabilities (e.g. in mobile devices, PCs) or inherently not trustworthy (e.g., cloud infrastructures) is a major challenge.

A promising starting point for solutions are the remote attestation capabilities offered by modern trusted hardware: computational platforms equipped with this technology can guarantee to a remote party various degrees of integrity for the software that it runs. For example the Trusted Platform Module (TPM) can provide certified measurements on the state of the platform and can be used to guarantee integrity of BIOS and boot code right before it is executed. More recent technologies (e.g., ARM's TrustZone and Intel's Software Guard Extension (SGX) [19]) have significantly expanded the scope and guarantees of trusted hardware. They offer the ability to run applications in "clean-slate" isolated execution environments (IEE) completely independent of anything else running on the processor; the desired attestation guarantees come from reports that are authenticated cryptographically.

A second major challenge is to provide security guarantees that go beyond heuristic arguments. Here, the established methodology is the "provable security" approach, which advocates carrying out the analysis of systems with respect to rigorously specified models that clarify the trust relations, the powers of the adversary and what constitutes a security breach. The approach offers well-established definitional paradigms for all basic primitives and some of the more used protocols. On the other hand, the success of applying this approach to new and more complex scenarios fundamentally hinges on one's ability to tame scalability problems, as models and proofs, even for moderate size systems, tend to be unwieldy. Some solutions to this issue exist in the form of compositional principles that, when incorporated as a native feature in the security abstractions, allow to establish the guarantees of larger systems from the guarantees on its components [9].

In this paper we take a provable security approach to protocols that rely on the IEE-capabilities of modern trusted hardware. It may be tempting to assume that, for the analysis of such protocols, designing security models is a simple matter of overlaying/merging the trust model induced by the use of such hardware over well-established security abstractions. If this were true, one could rely on established models and methodologies to perform the analysis. Unfortunately, this is not the case.

Consider the problem of secure outsourced computation of a program $P$ to a remote machine. The owner of $P$ wants 
to ensure that the (potentially malicious) remote platform does not tamper with the execution of the program and that it learns no information about the input/output (I/O) behavior of $P$. For remote machines with IEE capabilities, the following straightforward design has been informally proposed in the literature and should, intuitively, provide the desired guarantees. First, execute a key-exchange with a remote instance of an IEE; after the key exchange finishes, use the key thus established to send encrypted inputs to the IEE who can decrypt and pass them to $P$. The output returned by $P$ is encrypted within the IEE and sent to the user. The construction relies on standard building blocks (key-exchange, authenticated encryption) so the security of the overall design should be reducible to that of the key exchange and authenticated encryption, for which we already have widely-accepted security models and constructions.

We highlight two important issues that show that neither existent models nor existent techniques are immediately suitable for the analysis of IEE-based protocols in general, and for the protocol above in particular. The first is the concept of a party which is a key notion in specifying and reasoning about the security of distributed systems. Traditionally, one considers security (e.g., of a key-exchange) in a setting where there is a PKI and at least some of the parties (e.g., the servers) have associated public keys. Parties and their cryptographic material are then essentially the same thing for the purpose of the security analysis. In the context that we study, users (who wish to use trusted hardware) are not expected to have long term keys; furthermore, privacy considerations require that the cryptographic operations performed by the trusted hardware on remote machines should not allow one to track different instances - which makes long term cryptographic material inadequate as a technical anchor of a party's participation in such a protocol. Indeed, the desirable functionality for many usages of such systems is that the cryptographic material associated with a computation outsourcing protocol (both for local and remote parties) can be arbitrary and fixed on-the-fly, when the protocol is executed. An interesting problem is therefore how to define the security of outsourced computation in this setting, and how to rely on the asymmetry afforded by the trust model specific to IEE systems to realise it.

The second issue is composability. In the protocol for outsourced computation that we present above, one might be led to think that security simply follows if the keyexchange is secure and the channel between the user and the IEE uses authenticated encryption (with appropriate replay protection): if the only information passed from the key exchange to the channel is the encryption key, then one can design and analyze the two parts separately. While in more standard scenarios this may be true, reliance on the IEE breaks the independence assumption that allows for composability results: what the specification above hides is that the code run by the IEE (i.e., the program for keyexchange, the one for the secure channels and the program that they protect) needs to be loaded at once, or else no isolation guarantees are given by the trusted hardware. ${ }^{1}$ This means that the execution of the different parts of the program is not necessarily independent, as they unavoidably share the state of the IEE. An important question is therefore whether the above intuitive construction is sound, and under which conditions can one use it to perform IEE-enabled outsourcing of computation.

To summarise the above discussion, two remarks help motivate the work in this paper. Protocols relying on IEE are likely to be deployed in applications with stringent security requirements, so ensuring that they fall under the scope of the provable security approach is important. Moreover, such applications are likely to be complex: inherently, they involve communication between remote parties, incorporate diverse code executed at different levels of trust, and rely on multiple cryptographic primitives and protocols as building blocks (see, for example, the One-time password protocol based on SGX[19]). However, we have concluded that key aspects of existing cryptographic models do not naturally translate to this new setting and, perhaps more worryingly, that the type of compositional reasoning enabled by such cryptographic models is clearly unsuitable for protocols that rely on IEE.

OUR APPROACH. We will first outline the high-level decisions that underly our approach, and then describe our technical contributions more in detail. We use SGX and TrustZone as inspiration, but we do not hardwire our models to a specific platform, in order to ensure that the scope of our work encompasses other similar technologies. Instead, we use an abstract notion of a machine which captures the relevant aspects that such platforms offer: their capability to run processes with isolation guarantees and the ability to directly use secure cryptography without going through potentially untrusted software. Additionally, we target our effort on the combination of remote attestation and keyexchange protocols. The former is the raison d'être for trusted hardware, while the latter is the natural building block towards adding secrecy guarantees for code running under the protection of IEEs (per our example above).

As explained above, one challenge we set out to address is to incorporate into our approach a new form of compositional reasoning that permits dealing with potentially shared state between all the code that is loaded into an IEE, which means that a-priori there are no guarantees of independence between the executions of different cryptographic primitives

1. Intuitively, mechanisms such as SGX and TrustZone are designed to protect and provide attestation guarantees over monolithic pieces of software, which must be fixed when an IEE is created and are identified using a fingerprint of the code. To go around this restriction and compose multiple programs, one has two options: i. to build a single composed program and load it in its entirety into an IEE (this is the approach we follow in this paper); or ii. to use multiple IEEs to host the various programs, and employ cryptographic protocols to protect the interactions between them using the (potentially malicious) host operating system as a communications channel. We note that this latter option would again lead to loading composed programs into each IEE, since cryptographic code would need to be added to each of the individual programs, and this would then lead to the same problem that we intend to solve with the framework we propose in this paper. 
that one could incorporate into the same program. Indeed, at the very least, the reporting mechanisms for the IEE will refer to the code of the full program, which immediately constrains modular reasoning - a crucial tool to enable scalability. We sidestep this problem by providing definitions (for both syntax and security) that are compositionaware: they explicitly assume that the code loaded into an IEE may result from the composition of multiple programs.

Contributions. We focus on the interplay between composition and attestation in hardware-based settings. In particular, we concentrate on a pervasive use case: attestation is often used to protect a security-critical part of the code $\left(P^{*}\right.$ in our setting) whereas the remaining code $Q$ does not need attestation: it can rely on guarantees established by $P^{*}$ (e.g. an authenticated secret key), and can therefore be much more efficient. As explained above and in footnote 1, the stateful nature of the execution environment does not allow for independent analysis of these two components and new techniques for rigorous validation are needed.

Our starting point is therefore a program $Q$ that a local user wishes to outsource to a remote machine. Such a program will need to be transformed (compiled in the cryptographic sense of the word) into another program that, intuitively, will result from the composition of a handshake/bootstrapping procedure $P^{*}$ that will establish a secure channel with the IEE in which program $Q$ will be executed, and an instrumented version of $Q$, say $Q^{*}$, that uses the aforementioned secure channel to ensure that $Q$ is indeed securely executed.

Our approach to formalising and realising this composition pattern has three main stepping-stones: $i$. we introduce attested computation as the formalisation of the raw guarantees provided by IEEs with cryptographic functionalities; ii. we show how a passively secure key exchange can be efficiently combined with an attested computation scheme to obtain the bootstrapping procedure $P^{*}$ referred above; and iii. we rely on our composition-aware formalisation to show that by instrumenting program $Q^{*}$ using standard cryptographic techniques one achieves secure outsourced computation. Details follow.

Attested Computation. Our first contribution is a formal treatment of IEE-based remote attested computation. We consider a setting where a user wishes to remotely execute a program $P$ and rely on the cryptographic infrastructure available within the IEE to attest that some incarnation $P^{*}$ of this program is indeed executing within an IEE of a specific remote machine (or group of machines). We provide a general solution to this problem in the form of a new cryptographic concept called an attested computation. We formalize two core guarantees.

First, we demand that the user's local view of the execution is "as expected", i.e., that the I/O behavior that is reconstructed locally corresponds to an honest execution of $P$. The second guarantee is more subtle and requires that such an execution has actually occurred in an IEE within a specific remote platform; in other words, the attested computation client is given the assurance that its code is being run in isolation (and displays a given I/O behaviour) within a prescribed remote physical machine (or group of machines) associated with some authenticated public parameters. This latter guarantee is crucial for bootstrapping the secure outsourcing of code: consider for example $P$ to be a key-exchange protocol, which will be followed by some other program that relies on the derived key. It must be the case that, at the end of the key exchange, the remote state of the key-exchange is protected by an IEE.

The second guarantee we demand from attested computation follows easily from the previous observation. If the attested program keeps sensitive information in its internal state (which is not revealed by its I/O behaviour as in the case of a key exchange protocol) then execution within the remote IEE should safeguard its internal state. If this were not the case, we would again run into problems when trying to compose $P^{*}$ with some program that relies on the security properties of $P$. We therefore exclude attested computation schemes where the instrumented program $P^{*}$ might leak more information in its $\mathrm{I} / \mathrm{O}$ behaviour than $P$ itself by introducing the notion of minimal leakage, which essentially states that the I/O of an attested program does not leak any information beyond what is unavoidably leaked by an honest execution.

Finally, we provide a scheme for attested computation that relies on a remote machine offering a combination of symmetric authentication and digital signatures (a capability similar to what SGX provides) and show that our scheme is secure in the sense that we define.

KEY-EXCHANGE FOR ATTESTED COMPUTATION. On its own, attested computation only provides integrity guarantees: the I/O behavior of the outsourced code is exposed to untrusted code in the remote machine on which it is run. The natural solution to the problem is to establish a secure communication channel with the IEE via a key-exchange protocol. It is unclear, however, how the standard security models for key exchange protocols map into the attested computation scenario, and how existing constructions for secure key-exchange fare in the novel scenario that we study. Indeed, for efficiency reasons one should use a key exchange protocol that is just strong enough to achieve this goal.

To clarify this issue we formalize the notion of keyexchange for attested computation. The name that we propose is intentional: key-exchange protocols as used in the our context differ significantly in the syntax and security models from their more traditional counterparts. For example, our syntax reflects that the code of the key-exchange is not fixed a-priori: a user can set parameters both for the component to be run locally and for the one to be executed within the IEE. This allows a user to hardwire in the code to be run remotely a new nonce (or as in our examples some cryptographic public key for which it knows the secret key).

As explained above, the notion of party in the context of attested computation needs to be different from that adopted by traditional notions of secure key-exchange. Our solution is to rely on the trust model specific to IEE settings: we can assign some arbitrary strings as identifiers for the users of the local machine, and we allow these users to specify 
arbitrary strings as identifiers for the remote code (a secure instantiation would require that this identifier corresponds to some cryptographic material possibly generated on the fly as explained above). We then adapt the execution model and definitions for key-exchange for the modified syntax and the new notion of communicating parties to reflect the expected guarantees: different local and remote sessions agree on each other's identifiers, derive the same key and the key is unknown to the adversary. One crucial aspect of our security model for key-exchange is that it explicitly accounts for the fact that the remote process will be run under attestation guarantees, which maps to a semi-active adversarial environment.

To improve usability of our notion of secure keyexchange for attested computation we provide two results. The first result simplifies the design of such protocols. Here, we show a generic construction that combines a keyexchange protocol that is passively secure and a standard signature scheme to derive a (potentially very efficient) key exchange protocol for attestation. The second result simplifies reasoning about the composition of a key-exchange for attestation with an arbitrary protocol that relies on the agreed key. Specifically, we provide a utility theorem which specifically states what composition guarantees one gets for an arbitrary program $Q$ that is run within a remote IEE and relies on a shared key that was established via the attested computation of a key exchange protocol that satisfies our tailored definition.

SECURE OUtSOURCED COMPUTATION. The last layer in our framework is a formalisation of secure outsourced computation, the principal motivating use-case for our approach. We provide syntax and two security notions for a secure outsourced computation protocol, one for authenticity and the second one for the privacy of the I/O of the outsourced program. We then prove that the construction that combines a key-exchange for attested computation with an authenticated symmetric encryption scheme and replay protection gives rise to a scheme for secure outsourced computation. We present our result as a general formalisation (i.e., not application specific) of the intuition that by relying on more powerful hardware assumption such as those offered by SGX, one can indeed efficiently achieve a well-defined notion of secure outsourced computation that simultaneously offers verifiability and privacy. The proof of this result crucially relies on the utility theorem we defined for the combination of attested computation with key exchange.

\section{Other Related work}

Work that looks at provable security of realistic protocols that use trusted hardware-based protocols has developed around the protocols offered by the Trusted Platform Module (TPM) [6], [29], [7], [13], [12]. However, the functionality and efficiency of the protocols offered by the TPM makes them more suitable for static attestation (i.e., ensuring integrity of programs right before they are executed). Run-time guarantees, like those that we study here are in principle possible but cumbersome to obtain.

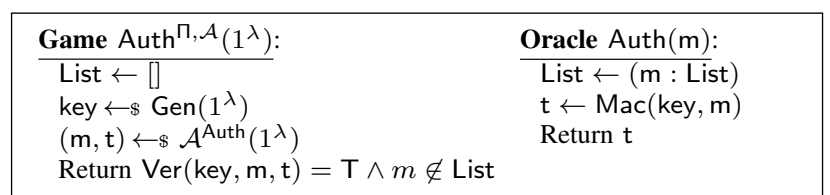

Figure 1: Game defining the security of a MAC scheme $\Pi$.

Linking attestation guarantees provided by the TPM with those offered by a secure channel onto a remote machine had been studied before but only informally [17]. Although more rigorous approaches used to analyze attestation guarantees for protocols based on the TPM exist, they use more abstract models (with weaker guarantees) [28], [11].

Another related line of research leverages the trusted hardware to bootstrap entire platforms for secure software execution (e.g. Flicker [23], Trusted Virtual Domains [10], Haven [3]). These are large systems that are currently outside the scope of provable-security techniques. Smaller protocols which solve specific problems (secure disk encryption [24], one-time password authentication [19] outsourced Map-Reduce computations [27], Secure Virtual Disk Images [14], secure embedded devices [25], [22]) are more susceptible to rigorous analysis. Although some protocols (e.g., those of Hoekstra et al. [19]) come only with intuition regarding their security, others - most notably those by Schuster et. al [27] which uses SGX platforms to outsource map-reduce computation - come with a proof of security. The constructions in that paper are close to those that we abstract and analyze here.

Our construction of secure outsourced computation can be seen as a solution to the problem of verifiable computation as specified by [15]. That rich line of work concentrates on the much harder problem of providing cryptoonly solutions and usually gives up privacy for efficiency and verifiability (e.g., [5], [16], [26]). Here, we show how to obtain a reasonably efficient and secure system with technology that is likely to be deployed in the near future.

\section{Preliminaries}

Message Authentication Codes. A message authentication code scheme $\Pi$ is a triple of PPT algorithms (Gen, Auth, Ver). On input $1^{\lambda}$, where $\lambda$ is the security parameter, the randomized key generation algorithm returns a fresh key. On input key and message $m$, the deterministic MAC algorithm Auth returns a tag t. On input key, $m$ and $t$, the deterministic verification algorithm Ver returns $T$ or $\mathrm{F}$ indicating whether $\mathrm{t}$ is a valid MAC for $\mathrm{m}$ relative to key. We require that, for all $\lambda \in \mathbb{N}$, all key $\in\left[\operatorname{Gen}\left(1^{\lambda}\right)\right]$ and all $\mathrm{m}$, it is the case that $\operatorname{Ver}($ key, $\mathrm{m},($ Auth $($ key, $\mathrm{m})))=\mathrm{T}$.

We use the standard notion of existential unforgeability for MACs [4]. We say that $\Pi$ is existentially unforgeable if $\operatorname{Adv}_{\mathcal{A}, \Pi}$ Auth $(\lambda)$ is negligible for every ppt adversary $\mathcal{A}$, where advantage is defined as the probability that the game in Figure 1 returns $\mathrm{T}$.

Digital Signature Schemes. A signature scheme $\Sigma$ is a triple of PPT algorithms (Gen, Sign, Vrfy). On input 


\begin{tabular}{|ll|}
\hline Game $\mathrm{UF}^{\Sigma, \mathcal{A}}\left(1^{\lambda}\right):$ & $\operatorname{Oracle} \operatorname{Sign}(\mathrm{m}):$ \\
\cline { 2 - 2 } List $\leftarrow]$ & $\sigma \leftarrow \operatorname{Sign}(\mathrm{sk}, \mathrm{m})$ \\
$(\mathrm{pk}, \mathrm{sk}) \leftarrow \$ \operatorname{Gen}\left(1^{\lambda}\right)$ & $\operatorname{Return} \sigma$ \\
$(\mathrm{m}, \sigma) \leftarrow \$ \mathcal{A}^{\operatorname{Sign}}\left(1^{\lambda}, \mathrm{pk}\right)$ & \\
Return $\operatorname{Vrfy}(\mathrm{pk}, \mathrm{m}, \sigma)=\mathrm{T} \wedge m \notin$ List & \\
\hline
\end{tabular}

Figure 2: Game defining the security of a signature scheme $\Sigma$.

$1^{\lambda}$, where $\lambda$ is the security parameter, the randomized key generation algorithm returns a fresh key pair (pk, sk). On input secret key sk and message $m$, the possibly randomized signing algorithm Sign returns a signature $\sigma$. On input public key $\mathrm{pk}, \mathrm{m}$ and $\sigma$, the deterministic verification algorithm Vrfy returns $\mathrm{T}$ or $\mathrm{F}$ indicating whether $\sigma$ is a valid signature for $\mathrm{m}$ relative to pk. We require that, for all $\lambda \in \mathbb{N}$, all $(\mathrm{pk}, \mathrm{sk}) \in\left[\operatorname{Gen}\left(1^{\lambda}\right)\right]$ and all $\mathrm{m}$, it is the case that $\operatorname{Vrfy}(\mathrm{pk}, \mathrm{m},(\operatorname{Sign}(\mathrm{sk}, \mathrm{m})))=\mathrm{T}$.

We use the standard notion of existential unforgeability for signature schemes [18]. We say that $\Sigma$ is existentially unforgeable if $\operatorname{Adv}_{\mathcal{A}, \Sigma}^{\mathrm{UF}}(\lambda)$ is negligible for every ppt adversary $\mathcal{A}$, where advantage is defined as the probability that the game in Figure 2 returns $T$.

PASSIVEly SECURE KEY EXCHANGe. We define a form of key exchange protocol that does not rely on long term secret/state or global setup and for which we require weak security guarantees (essentially security against a passive adversary); the classical Diffie-Hellman key exchange is a standard example. Later we show how, combined with attestation, such protocols yield secure key-exchange when facing active adversaries. A key exchange protocol is therefore defined by a single ppt algorithm $\Pi$ used by communicating parties. When analysing the security and correctness of the protocol we will consider that each party with identifier id can execute several instances of the protocol with different parties. Throughout the paper we let identifiers be arbitrary strings, which will be given meaning by the higherlevel application relying on the protocol under analysis. For $s \in \mathbb{N}$, we write $\Pi_{\text {id }}^{s}$ for the $s$ instance of party id. We assume that each instance maintains variables st, $\delta$, key which record respectively, local state information, the state of the key (derived, accept, reject or $\perp$ ) and the value of the key. In addition, we assume variables for the role $\rho$ of the session (initiator or responder), the party identifier of the owner of the session oid and that of its partner pid and a session identifier sid. We require that key $=\perp$ unless $\delta \in\{$ derived, accept $\}$, that oid, $\rho$, sid, key are only assigned once during the entire execution of the protocol (the first two when the session is initialized). We denote running $\Pi$ with message $\mathrm{m}$, role $\rho$ and state st to produce $\mathrm{m}^{\prime}$ and the updated state $\mathrm{st}^{\prime}$ by $\left(\mathrm{m}^{\prime}, \mathrm{st}^{\prime}\right) \leftarrow \$ \Pi\left(1^{\lambda}, \mathrm{m}\right.$, id, $\left.\rho, \mathrm{st}\right)$, and will omit the security parameter input throughout the paper for the sake of compactness. A key exchange protocol is correct if, after a complete (honest) run between two participants with complementary roles, both reach the accept state, both derive the same key and session identifier, and both obtain correct partner identifier strings.

We will consider key exchange schemes that are secure against passive adversaries. Our adopted security notion is a restriction of the scenario considered in [21] that excludes corruptions. ${ }^{2}$ The execution model considers an adversary, which is run on the security parameter, and which can interact with the following oracles whose behaviour depends on a secret sampled bit $b$ and a shared list of pairs of keys fake, which is initially empty:

- Execute $(i, j)$ runs a new instance of the protocol between distinct parties $i$ and $j$. It then checks if the key derived for the executed session exists in list fake. If not, it generates a new key* uniformly at random, and adds (key, key $\left.{ }^{*}\right)$ to the list. Finally, it outputs the transcript of the protocol execution and the session identifier associated with it.

- Reveal $(i, s)$ outputs the session key key of $\Pi_{i}^{s}$.

- Test $(i, s)$ will return $\perp$ if $\delta_{i}^{s} \neq$ accept (i.e. if no execute query actually created such a session). Otherwise, if $b=0$ it outputs the key associated with $\Pi_{i}^{s}$. If $b=1$, it searches for the key associated with $\Pi_{i}^{s}$ in list fake and returns the associated key*.

When the adversary terminates interacting with the oracles, it will eventually output a bit $b^{\prime}$ which represents his guess on what the challenge bit $b$ is.

We define entity authentication following [8], and observe that in the case of passively secure key exchange, this is essentially a correctness property. First we introduce a notion of partnering, which informally states that two oracles which have derived keys are partners if they share the same session identifier. The definition makes use of the following predicate on two instances $\Pi_{i}^{s}$ and $\Pi_{j}^{t}$ holding states $\left(\mathrm{st}_{i}^{s}, \delta_{i}^{s}, \rho_{i}, \operatorname{sid}_{i}^{s}, \operatorname{pid}_{i}^{s}, \operatorname{key}_{i}^{s}\right)$ and $\left(\mathrm{st}_{j}^{t}, \delta_{j}^{t}, \rho_{j}, \operatorname{sid}_{j}^{t}, \operatorname{pid}_{j}^{t}, \operatorname{key}_{j}^{t}\right)$, respectively:

$\mathrm{P}\left(\Pi_{i}^{s}, \Pi_{j}^{t}\right)=\left\{\begin{array}{l}\mathrm{T} \text { if } \operatorname{sid}_{i}^{s}=\operatorname{sid}_{j}^{t} \wedge \delta_{i}^{s}, \delta_{j}^{t} \in\{\text { derived, accept }\} \\ \mathrm{F} \text { otherwise. }\end{array}\right.$

Definition 1. Two players $\Pi_{i}^{s}$ and $\Pi_{j}^{t}$ are partnered if $\mathrm{P}\left(\Pi_{i}^{s}, \Pi_{j}^{t}\right)=\mathrm{T}$.

Our authentication notion relies on three further definitions, which demand that partnerings will need to be valid, confirmed and unique. In short, these three requirements ensure that any instance that accepts has a partner, that this partner is unique and that partners share the same key.

Definition 2 (Valid Partners). A protocol $\Pi$ ensures valid partners if the bad event notval does not occur, where notval is defined as follows:

$$
\begin{aligned}
& \exists \Pi_{i}^{s}, \Pi_{j}^{t} \text { s.t. } \mathrm{P}\left(\Pi_{i}^{s}, \Pi_{j}^{t}\right)=\mathrm{T} \wedge \\
& \quad\left(\operatorname{pid}_{i}^{s} \neq \operatorname{oid}_{j}^{t} \vee \operatorname{pid}_{j}^{t} \neq \operatorname{oid}_{i}^{s} \vee \rho_{i}=\rho_{j} \vee \operatorname{key}_{i}^{s} \neq \operatorname{key}_{j}^{t}\right) .
\end{aligned}
$$

2. The trust model we consider for attested computation excludes corruptions for the sake of simplicity, but all our results can be extended to consider that possibility. Having said that, it seems reasonable to exclude the possibility of isolated executed environment breach based on a hardware assumption. The possibility of local machine corruption should, however, be considered. 
Definition 3 (Confirmed Partners). A protocol $\Pi$ ensures confirmed partners if the bad event notconf does not occur, where notconf is defined as follows:

$$
\exists \Pi_{i}^{s} \quad \text { s.t. } \quad \delta_{i}^{s}=\text { accept } \wedge \forall \Pi_{j}^{t}, \mathrm{P}\left(\Pi_{i}^{s}, \Pi_{j}^{t}\right)=\mathrm{F} .
$$

Definition 4 (Unique Partners). A protocol $\Pi$ ensures unique partners if the bad event notuni does not occur, where notuni is defined as follows:

$$
\begin{aligned}
& \exists \Pi_{i}^{s}, \Pi_{j}^{t}, \Pi_{k}^{r} \text { s.t. } \\
& \quad(j, \mathrm{t}) \neq(k, r) \wedge \mathrm{P}\left(\Pi_{i}^{s}, \Pi_{j}^{t}\right)=\mathrm{T} \wedge \mathrm{P}\left(\Pi_{i}^{s}, \Pi_{k}^{r}\right)=\mathrm{T}
\end{aligned}
$$

Intuitively, we will consider that an adversary violates two-sided entity authentication if he can lead an instance of an honest party running the protocol to accept, and in doing that cause one of the bad events notval, notconf, notuni.

We are now ready to present the security notion we will be using for key exchange protocols. To exclude breaks via trivial attacks, we define legitimate adversaries as those who ensure the following freshness criteria is satisfied for his Test $(i, s)$ queries: i. Reveal $(i, s)$ was not queried; and ii. for all $\Pi_{j}^{t}$ such that $\mathrm{P}\left(\Pi_{i}^{s}, \Pi_{j}^{t}\right)=\mathrm{T}$, Reveal $(j, t)$ was not queried. We only consider experiments in which the adversary is found to be legitimate and define the winning event guess to be $b=b^{\prime}$ at the end of the experiment.

Definition 5. A protocol $\Pi$ is passively secure if, for any legitimate ppt adversary: 1. the adversary violates two-sided entity authentication with negligible probability; and 2. its key secrecy advantage $2 \cdot \operatorname{Pr}[$ guess ] -1 is negligible.

\section{IEEs, Programs, and Machines}

ISOLATED EXECUTION ENVIRONMENTS. At the high-level, an IEE can be seen as an idealised random access machine running some fixed program $P$, whose behaviour can only be influenced via a well-specified interface that permits passing inputs to the program, and receiving its outputs. Intuitively, an IEE gives the following security guarantees, which we will formalise later in this section. The I/O behaviour of a process running in an IEE is determined by the program it is running, the semantics of the language in which the program is written, and the inputs it receives. This means, in particular, that there is strict isolation between processes running in different IEEs (and any other program running on the machine). Furthermore, the only information that is revealed about a program running within an IEE is contained in its input-output behaviour (which in most hardware systems is simply shared memory between the protected code and the untrusted software outside).

We emphasize that our notion of a machine is intended to be inclusive of any hardware platform that supports some form of isolated execution. For this reason, the syntax of this abstraction is minimalistic, so that it can be restricted/extended to capture the specific guarantees awarded by different concrete hardware architectures, including TPM, TrustZone, SGX, etc. As an example, our "vanilla" machine supports an arbitrary number of IEEs, where programs can be loaded only once, and where multiple input/output interactions are allowed with the protected code. This is a close match to the SGX/TrustZone functionalities. However, for something like TPM, one could consider a restricted machine where a limited number of IEEs exist, with constrained input/output capabilities, and running specific code (e.g., to provide key storage). Similarly, we consider IEE environments where the underlying hardware is assumed to only keep benevolent state, i.e., state that cannot be used to introduce destructive correlations between multiple interactions with an IEE. Again, this closely matches what happens in SGX/Trustzone, but different types of state keeping could be allowed for scenarios where such correlations are not a problem or where they must be dealt with explicitly.

PROGRAMS. Implicit throughout the paper will be a programming language $\mathcal{L}$ in which programs are written. We assume that this language is used by all computational platforms, but we admit IEE-specific system calls giving access to different cryptographic functionalities. These are referred as the security module interface. An additional system call rand is also assumed to be present in all platforms, giving access to fresh random coins sampled uniformly at random. Language $\mathcal{L}$ is assumed to be deterministic modulo the operation of system calls. As mentioned above, it is important for our results that system calls cannot be used by a program to store additional implicit state that would escape our control. To this end, we impose that the results of system calls within an IEE can depend only on: i. an initially shared state that is defined when a program is loaded (e.g., the cryptographic parameters of the machine, and the code of the program); ii. the input explicitly passed on that particular call; and iii. fresh random coins. As a consequence of this, we may assume that system calls placed by different parts of a program are identically distributed, assuming that the same input is provided. This is particularly important when we consider program composition below.

A program $P$ must be written as a transition function, mapping bit-strings to bit-strings. Such functions take a current state st and an input $i$, and they will produce a new output $o$ and an updated state. We will refer to this as an activation and express it as $o \leftarrow P[\mathrm{st}](i)$. Unless otherwise stated, st will be assumed to be initially empty. We impose that every output produced by a program includes a Boolean flag finished that indicates whether the transition function will accept further input. The transition function may return arbitrary output until it produces an output where finished $=\mathrm{T}$, at which point it can return no further output or change its state. We extend our notation as $o \leftarrow P[\mathrm{st} ; r](i)$ to account for the randomness obtained via the rand system call as extra input $r$; and as $\left(o_{1}, \ldots, o_{n}\right) \leftarrow P[\mathrm{st} ; r]\left(i_{1}, \ldots, i_{n}\right)$ to represent a sequence of activations. We write $\operatorname{Trace}_{P[\mathbf{s t} ; r]}\left(i_{1}, \ldots, i_{n}\right)$ for the corresponding $\mathrm{I} / \mathrm{O}$ trace $\left(i_{1}, o_{1}, \ldots, i_{n}, o_{n}\right)$.

Program composition. Given two programs $P$ and $Q$, and a projection function between the internal states of the two programs $\phi$, we will refer to the sequential composition 
of the two programs as Compose ${ }_{\phi}\langle P, Q\rangle$. This is defined as a transition function $R$ that has two execution stages, which are signaled in its output via an additional stage bit. In the first stage, every input to $R$ will activate program $P$. This will proceed until $P$ 's last output indicates it has finished (inclusively). The next activation will trigger the start of the second stage, at which point $R$ initialises the state of $Q$ using $\phi\left(\mathrm{st}_{P}\right)$ before activating it for the first time. Additionally we require that a constant indicating the current stage (termination being counted as a third stage) is appended to any output of a composition. When dealing with such a composed program, we will denote by $\operatorname{ATrace}_{R[\mathrm{st} ; r]}\left(i_{1}, \ldots, i_{n}\right)$ the prefix of the trace that corresponds to the execution of $P$. Intuitively, this denotes the attested trace where only the initial part of the program must be protected via attestation.

MACHINES. A machine $\mathcal{M}$ is an abstract computational device that captures the resources offered by a real world computer or group of computers, whose hardware security functionalities are initialised by a specific manufacturer before being deployed, possibly in different end-users.

We will model machines via a simple external interface, which we see as both the functionality that higher-level cryptographic schemes can rely on when using the machine, and the adversarial interface that will be the basis of our attack models. Loosely speaking, this interface can be thought of as the ideal functionality that captures a system such as SGX [20]. The interface is as follows:

- $\operatorname{Init}\left(1^{\lambda}\right)$ is the global initialisation procedure which, on input the security parameter, outputs the global parameters prms. This algorithm represents the machine's hardware initialisation procedure, which is out of the user's and the adversary's control. Intuitively, it initialises the internal security module, the internal state of the remote machine and returns any public cryptographic parameters that the security module releases. We emphasize that the global parameters of machines are the only pieces of information that are assumed to be authenticated using external mechanisms (such as a PKI) in the entire paper.

- $\operatorname{Load}(P)$ is the IEE initialisation procedure. On input a program/transition function $P$, the machine produces a fresh handle hdl, creates a new IEE with handle hdl, loads $P$ into the new IEE and returns hdl. The machine interface does not provide direct access to either the internal state of an IEE nor to its randomness input. This means that the only information that is leaked about internal state and randomness input is that revealed (indirectly) via the outputs of the program.

- Run (hdl, $i$ ) is the process activation procedure. On input a handle hdl and an input $i$, it will activate process running in isolated execution environment of handle hdl with $i$ as the next input. When the program/transition function produces the next output $o$, this is returned to the caller.

We define the $\mathrm{I} / \mathrm{O}$ trace $\operatorname{Trace}_{\mathcal{M}}(\mathrm{hdl})$ of a process hdl running in some machine $\mathcal{M}$ as the tuple $\left(i_{1}, o_{1}, \ldots, i_{n}, o_{n}\right)$ that includes the entire sequence of $n$ inputs/outputs resulting from all invocations of the Run procedure on hdl; Program ${ }_{\mathcal{M}}(\mathrm{hdl})$ is the code (program) running inside

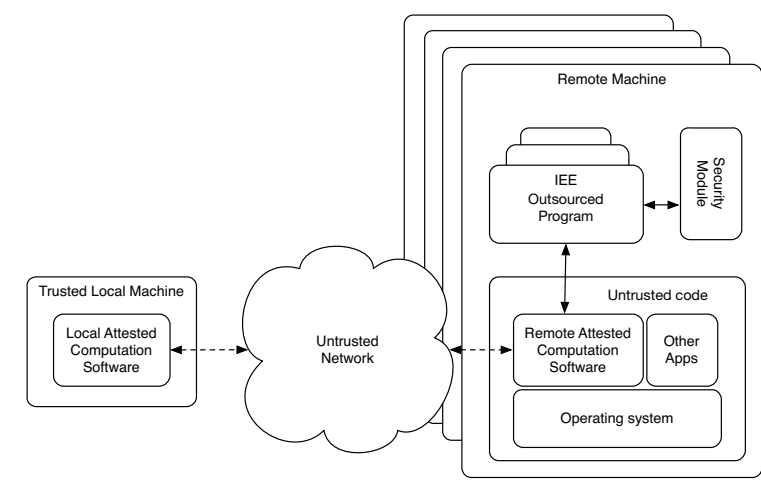

Figure 3: Attested Computation scenario.

the process with handle hdl; Coins $\mathcal{M}(\mathrm{hdl})$ represents the coins given to the program by the rand system call; and State $_{\mathcal{M}}(\mathrm{hdl})$ is the internal state of the program. Finally, we will denote by $\mathcal{A}^{\mathcal{M}}$ the interaction of some algorithm with a machine $\mathcal{M}$, i.e., having access to the Load and Run oracles defined above.

\section{Attested Computation}

We now formalise a cryptographic primitive that aims to address the remote execution, i.e., outsourcing, of programs as illustrated in Figure 3. In this setting, a user running software in a trusted local machine wishes to use an untrusted network to access a pool of remote machines with IEE facilities. The remote machines will be running generalpurpose operating systems and other untrusted software. The goal of the user is to run a specific program $P$ within an IEE in one of the remote machines, and to obtain assurance that, not only the program is indeed executing there, but also that it is displaying a particular I/O behaviour.

SyntaX. An Attested Computation (AC) scheme is defined by the following algorithms:

- Compile(prms, $P, \phi, Q)$ is the program compilation algorithm. On input global parameters for some machine $\mathcal{M}_{R}$, and programs $P$ and $Q$, whose composition under projection function $\phi$ will be outsourced, it will output program $R^{*}$, together with an initial (possibly empty) state st for the verification algorithm. This algorithm is run locally. $R^{*}$ is the code to be run as an isolated process in the remote machine. Intuitively, $P$ is the initial part of the remote code that requires attestation guarantees, whereas $Q$ is any subsequent code that may be remotely executed (generally leveraging the security guarantees that have been bootstrapped using the initial attested execution).

- Attest(prms, hdl, $i$ ) is the attestation algorithm. On input global parameters for $\mathcal{M}_{R}$, a process handle hdl and an input $i$, it will use the interface of $\mathcal{M}_{R}$ to obtain attested output $o^{*}$. This algorithm is run remotely, but in an unprotected environment: it is responsible for interacting with the isolated process running $R^{*}$, providing it with inputs and recovering the (possibly attested) outputs that should be returned to the local machine. 
- Verify (prms, $i, o^{*}$, st) is the (stateful) output verification algorithm. On input global parameters for $\mathcal{M}_{R}$, an input $i$, a (possibly attested) output $o^{*}$ and some state st, it will produce an output value $o$ and an updated state, or the failure symbol $\perp$. This failure symbol is encoded so as to be distinguishable from a valid output of a program, resulting from a successful verification.This algorithm is run locally on claimed outputs from the Attest algorithm.

In Figure 3, the local attested computation software block corresponds to Compile (one initial usage per program) and Verify (one usage per incoming attested output), whereas the remote attested computation software block corresponds to Attest (one usage per remote program activation, i.e. per I/O transition). The above syntax can be naturally extended to accommodate the simultaneous compilation of multiple input programs and/or the possibility that Compile may generate multiple output programs. This would allow us to capture, e.g., map/reduce applications such as those described in [27].

CORRECTNESS. Intuitively, an AC scheme is correct if, for any given programs $P$ and $Q$ and assuming an honest execution of all components in the scheme, both locally and remotely, the local user is able to accurately reconstruct a view of the $I / O$ sequence that took place in the remote environment. The following definition formalizes the notion of a local user correctly remotely executing program $P$ using attested computation.

Definition 6. An Attested Computation scheme AC is correct if, for all $\lambda$, and all adversaries $\mathcal{A}$, the experiment in Figure 4 (top) always returns $\mathrm{T}$.

The adversary in this correctness experiment definition is choosing inputs, hoping to find a sequence that causes the attestation protocol to behave inconsistently with respect to the semantics of $P$ (when these are made deterministic by hardwiring the same random coins used remotely). We use this approach to defining correctness because it makes explicit what is an honest execution of an attested computation scheme, when compared to the security experiment introduced next.

Structural PRESERVATION. Since we are dealing with composed programs, we extend the correctness requirements on attested computation schemes to preserve the structure of the input program $(P, \phi, Q)$, and to modify only the part of the code that will be attested. Formally, we impose that, given any program $P$, there exists a (unique) compiled program $P^{*}$, such that, for any mapping function $\phi$ and any program $Q$, we have that Compose $_{\phi}\left\langle P^{*} ; Q\right\rangle=$ Compile $(P, \phi, Q)$.

SECURITY. Security of an attested computation scheme imposes that an adversary with absolute control of the remote machine cannot convince the local user that some arbitrary remote execution of a program $P$ has occurred, when it has not (nothing is said about the subsequent remote execution of program $Q$ ). Formally, we allow the adversary to freely interact with the remote machine, whilst providing a sequence of (potentially forged) attested outputs. The ad- versary wins if the local user reconstructs an execution trace without aborting (i.e., all attested outputs must be accepted by the verification algorithm) and one of two conditions occur: i. the execution trace that is validated by Verify is inconsistent with the semantics of $P$ (in which case an adversary would be able to convince the local user of an I/O sequence that could not possibly have occurred!); or ii. there does not exist a remote process hdl ${ }^{*}$ exhibiting a consistent execution trace (in which case, the adversary would be able to convince the local user that a process running $P$ was executing in the remote machine, when it was not).

Since the adversary is free to interact with the remote machine as it pleases, we can not hope to prevent it from appending arbitrary inputs to the trace of any remote process, while refusing to deliver all of the resulting attested outputs to the local user. This justifies the winning condition in our security game referring to a prefix of the trace in the remote machine, rather than imposing trace equality. Indeed, the definition's essence is to impose that the locally recovered trace and the remote trace share a common prefix $(\sqsubseteq)$, which exactly corresponds to the part of the source program's behaviour that should be protected by attestation.

Formally, we need to account for the fact that the actual I/O sequence of the remote program includes more information than that of $R$, e.g., to allow for the cryptographic enforcement of security guarantees. Our definition is parametrised by a Translate algorithm that permits formalising this notion of semantic consistency. Another way to see Translate(prms, ATrace $\mathcal{M}_{R}\left(\right.$ hdl $\left.\left.^{*}\right)\right)$ is as a trace translation procedure associated with a given $\mathrm{AC}$ scheme, which maps remote traces into traces at the source level.

Definition 7. An attested computation scheme is secure if there exists an efficient deterministic algorithm Translate s.t., for all ppt adversaries $\mathcal{A}$, the probability that experiment in Figure 4 (bottom) returns $\mathrm{T}$ is negligible.

We note that the adversary loses the game as long as there exists at least one remote process that matches the locally reconstructed trace. This should be interpreted as the guarantee that IEE resources are indeed being allocated in a specific remote machine to run at least one instance of the remote program (note that if the program is deterministic, many instances could exist with exactly the same I/O behaviour, which is not seen as a legitimate attack).

Minimum LeAKAGE. From the discussion above, an AC scheme should guarantee that the I/O behaviour of the program in the remote machine includes at least the information required to reconstruct an hypothetical local execution of the source program. However, it is important to establish an additional restriction on what $\mathrm{AC}$ compilation actually does to a source program, to ensure that we are able to take advantage of this primitive to achieve more ambitious goals, namely to perform attestation of the remote execution of cryptographic code.

The following definition imposes that nothing from the internal state of the source programs (in addition to what is public, i.e. the code and I/O sequence) is leaked in the trace of the compiled program when it is remotely executed. 


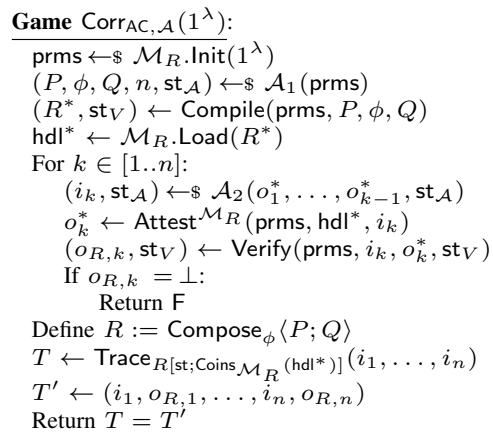

Figure 4: Games defining the correctness (top) and security (bottom) of an AC scheme.

\begin{tabular}{|c|c|}
\hline $\begin{array}{l}\text { Game Leak-Real }{ }_{\mathrm{AC}, \mathcal{A}}\left(1^{\lambda}\right): \\
\text { PrgList } \leftarrow[] \\
\text { prms } \leftarrow \$ \mathcal{M}_{R} \cdot \operatorname{lnit}\left(1^{\lambda}\right) \\
b \leftarrow \$ \mathcal{A}^{\mathcal{O}}(\mathrm{prms}) \\
\text { Return } b\end{array}$ & $\begin{array}{l}\text { Oracle Compile }(P, \phi, Q): \\
\left(R, \text { st }_{V}\right) \leftarrow \text { Compile }(\text { prms }, P, \phi, Q) \\
\text { PrgList } \leftarrow R: \text { PrgList } \\
\text { Return } R\end{array}$ \\
\hline$\frac{\text { Oracle } \operatorname{Load}(R):}{\text { Return } \mathcal{M}_{R} \cdot \operatorname{Load}(R)}$ & $\frac{\text { Oracle Run }(\text { hdl, } i):}{\text { Return } \mathcal{M}_{R} \cdot \operatorname{Run}(\text { hdl, } i)}$ \\
\hline
\end{tabular}

\begin{tabular}{|c|c|}
\hline \multicolumn{2}{|c|}{ Game Leak-Ideal $_{\mathrm{AC}, \mathcal{A}, \mathcal{S}}\left(1^{\lambda}\right)$ : Oracle Compile $(P, \phi, Q)$ : } \\
\hline PrgList $\leftarrow[]$ & $\left(R\right.$, st $\left._{V}\right) \leftarrow$ Compile $($ prms $, P, \phi, Q)$ \\
\hline List $\leftarrow[]$ & PrgList $\leftarrow(P, \phi, Q, R):$ PrgList \\
\hline hdl $\leftarrow 0$ & Return $R$ \\
\hline$($ prms, st $\mathcal{S}) \leftarrow \$ \mathcal{S}_{1}\left(1^{\lambda}\right)$ & \\
\hline$b \leftarrow \$ \mathcal{A}^{\mathcal{O}}(\mathrm{prms})$ & Oracle Run(hdl, $i)$ : \\
\hline Return $b$ & $(R, \mathrm{st}) \leftarrow$ List $[\mathrm{hd} \mathrm{l}]$ \\
\hline & If $(P, \phi, Q, R) \in$ PrgList: \\
\hline Oracle $\operatorname{Load}(R)$ : & $R^{*} \leftarrow$ Compose $_{\phi}\langle P, Q\rangle$ \\
\hline hdl $\leftarrow$ hdl +1 & $o^{*} \leftarrow \$ R^{*}[\mathrm{st}](i)$ \\
\hline List $[$ hdl $] \leftarrow(R, \epsilon)$ & $\left(o, \mathrm{st}_{\mathcal{S}}\right) \leftarrow \$ \mathcal{S}_{2}\left(\mathrm{hdl}, P, \phi, Q, R, i, o^{*}, \mathrm{st}_{\mathcal{S}}\right)$ \\
\hline Return hdl & Else: $\left(o, \mathrm{st}, \mathrm{st}_{\mathcal{S}}\right) \leftarrow \$ \mathcal{S}_{3}\left(\mathrm{hdl}, R, i, \mathrm{st}, \mathrm{st}_{\mathcal{S}}\right)$ \\
\hline & List $[\mathrm{hdl}] \leftarrow(R, \mathrm{st})$ \\
\hline & Return $o$ \\
\hline
\end{tabular}

Figure 5: Games defining minimum leakage of an AC scheme.

Definition 8. Attested Computation scheme AC ensures security with minimal leakage if it is secure according to Definition 7 and there exists a ppt simulator $\mathcal{S}$ that, for every adversary $\mathcal{A}$, the following distributions are identical:

$$
\left\{\text { Leak-Real }_{\mathrm{AC}, \mathcal{A}}\left(1^{\lambda}\right)\right\} \approx\left\{\text { Leak-Ideal }_{\mathrm{AC}, \mathcal{A}, \mathcal{S}}\left(1^{\lambda}\right)\right\}
$$

where games Leak-Real $\mathrm{AC, \mathcal {A }}$ and Leak-Ideal $\mathrm{AC, \mathcal {A } , \mathcal { S }}$ are shown in Figure 5.
Notice that we allow the simulator to replace the global parameters of the machine with some value prms for which it can keep some trapdoor information. Intuitively this means that one can construct a perfect simulation of the remote trace by simply appending cryptographic material to the local trace. This property is important when claiming that the security of a cryptographic primitive is preserved when it is run within an attested computation scheme (one can simply reduce the advantage of an adversary attacking the attested trace, to the security of the original scheme using the minimum leakage simulator).

\section{Attested Computation à la SGX}

The remote attestation protocol we will consider is inspired in the Secure Guard Extensions (SGX) architecture proposed by Intel [1]. The main feature of this system is that the remote machine is equipped with a security module that manages both short-term and long-term cryptographic keys, with which it is capable of producing MACs that enable authenticated communication between various IEEs and digital signatures that can be publicly verified by anyone holding the (long-term) public key for that machine (or group of machines). We first formalise the operation of (a simplified version of) this security module.

SECURITY MODULE. The security module relies on a signature scheme $\Sigma=$ (Gen, Sign, Vrfy) and a MAC scheme $\Pi=$ (Gen, Mac, Ver), and it operates as follows:

- When the host machine is initialised, the security module generates a key pair (pk, sk) using $\Sigma$. Gen and a symmetric key key using $\Pi$.Gen. It also creates a special process running code $S^{*}$ (see below for a description of $S^{*}$ ) in an IEE with handle 0 . The security module then securely stores the key material for future use, and outputs the public key. In this case we will have that the output of $\mathcal{M}$.Init will be prms $=$ pk.

- The operation of IEE with handle 0 will be different from all other IEEs in the machine. Program $S^{*}$ will permanently reside in this IEE, and it will be the only one with direct access to both sk and key.

- The code of $S^{*}$ is dedicated to transforming messages authenticated with key into messages signed with sk. On each activation, it expects an input $(\mathrm{m}, \mathrm{t})$. It obtains key from the security module and verifies the tag using П.Ver(key, $t, m)$. If the previous operation was successful, it obtains sk from the security module, signs the message using $\sigma \leftarrow \Sigma \Sigma$.Sign $(\mathrm{sk}, \mathrm{m})$ and writes $\sigma$ to the output. Otherwise, it writes $\perp$ in the output.

- The security module exposes a single system call $\operatorname{mac}(\mathrm{m})$ to code running in all other IEEs. On such a request from a process running program $P$, the security module returns a MAC tag t computed using key over both the code of $P$ and the input message $(\mathrm{m})$.

We note that the operation of the security module allows any process to produce an authenticated message that can be validated by the special process running $S^{*}$ as coming from within another IEE in the same machine. 
We will assume that the message authentication code scheme $\Pi$ and the signature scheme $\Sigma$ satisfy the standard notions of correctness and existential unforgeability detailed in Section 3, and that the machine's public key is authenticated by some external PKI.

AtTested Computation SCHEME. We now define an AC scheme that relies on a remote machine supporting a security module with the above functionality. The operation of the various algorithms is intuitive, except for the fact that basic replay protection using a sequence number does not suffice to bind a remote process to a full trace, since the adversary could then run multiple copies of the same process and mix and match outputs from various traces. Instead, the remote process must commit to its entire trace whenever an attested output is produced. Details follow:

- Compile(prms, $P, \phi, Q)$ will generate a new program $R^{*}=$ Compose $_{\phi}\left\langle P^{*}, Q\right\rangle$ and output it along with the initial state of the verification algorithm $\left(R^{*},[], 1\right)$, where 1 is an indicator of the stage in which remote program $R^{*}$ is supposed to be executing. Program $P^{*}$ is instrumented as follows: it keeps a list ios of all the I/O pairs it has previously received and computed, i.e, its own trace; on each activation with input $i, P^{*}$ first computes $o \leftarrow \$ P\left[\mathrm{st}_{P}\right](i)$ and updates the list by adding a new $(i, o)$ pair; it then requests from the security module a MAC of the updated ios. Due to the operation of the security module, this will correspond to a tag $\mathrm{t}$ on the tuple $\left(R^{*}\right.$, ios); it finally outputs $\left(o, \mathrm{t}, R^{*}\right.$, ios $)$. We note that we include $\left(R^{*}\right.$, ios $)$ explicitly in the outputs of $R^{*}$ for clarity of presentation only. This value would be kept in an insecure environment by a stateful Attest program.

- Attest(prms, hdl, $i$ ) invokes $\mathcal{M}_{R}$.Run(hdl, $i$ ) using the handle and input value it has received. When the process produces an output $o$, Attest parses it into $\left(o^{\prime}, \mathrm{t}, R^{*}\right.$, ios $)$. It may happen that parsing fails, e.g., if $Q$ is already executing, in which case Attest simply produces $O$ as its own output. Otherwise, it uses $\mathcal{M}_{R} \cdot \operatorname{Run}\left(0,\left(R^{*}\right.\right.$, ios, $\left.\left.\mathrm{t}\right)\right)$ to convert the tag into a signature $\sigma$ on the same message. If this conversion fails, then Attest produces the original output $o$ as its own output. Otherwise, it outputs $\left(o^{\prime}, \sigma\right)$.

- Verify $\left(\right.$ prms, $i, o^{*},\left(R^{*}\right.$, ios, stage $\left.)\right)$ returns $o^{*}$ if stage $=$ 2. Otherwise, it first parses $o^{*}$ into $(o, \sigma)$, appends $(i, o)$ to ios, and verifies the digital signature $\sigma$ using prms and $\left(R^{*}\right.$, ios $\left.)\right)$. If parsing or verification fails, Verify outputs $\perp$. If not, then Verify will check if output $o$ indicates that program $P^{*}$ has finished. If so, it will update stage to value 2 . In any case, it terminates outputting $O$.

It is easy to see that our AC scheme is correct. We now analyse its security. Let Translate be the deterministic function that receives the machine parameters and a list of tuples of the form $\left(i,\left(o, \mathrm{t}, R^{*}\right.\right.$,ios $\left.)\right)$ and returns a list of pairs of the form $(i, o)$.

Theorem 1. The AC scheme presented above provides secure attestation if the underlying MAC scheme П and signature scheme $\Sigma$ are existentially unforgeable. Furthermore, it unconditionally ensures minimum leakage.
The proof of the following theorem can be found in the full version [2]. The intuition behind the proof of secure attestation is straightforward: since all attested outputs (i.e. those processed by Verify until st $P$. finished $=\mathrm{T}$ ) are bound to a full trace of the execution, all accepted messages that pass AC.Verify must terminate a prefix of a remote trace for some instance of $R^{*}$. The only case in which the adversary could win would be if the signature verification performed by Verify accepts a message that was never authenticated by an IEE running $R^{*}$. However, in this case, the adversary is either breaking the MAC (to dishonestly execute Attest) or breaking the signature (and forging attested outputs directly). The minimum leakage property can be proven by constructing the trivial simulator that generates the machine parameters itself, simulates the entire machine for nonattested processes, and attaches MACs to the source I/O traces of attested programs.

\section{AKE for Attested Computation}

An intermediate step in constructing high-level applications that rely on attested computation is the establishment of a secure communications channel with a process running a particular program inside an IEE in the remote machine. After such a channel has been established, standard cryptographic techniques can be used to ensure (in combination with the isolation provided by IEEs) the integrity and confidentiality of subsequent computations. In this section we will see how attested computation, in combination with a specific flavour of a key exchange protocol can be seen as a bootstrapping process for this scenario.

We first formalize the precise requirements for a key exchange protocol that can be used in this setting (we call this authenticated key exchange for attested computation) and show how a simple transformation can be used to construct such protocols from any passively secure key exchange protocol. Later on we present a utility theorem that precisely describes what it means to use attested computation and a suitable key exchange protocol to establish a secure channel with an arbitrary remote program.

Definitions. Syntax. A Key Exchange for Attested Computation (AttKE) protocol is defined by the following pair of algorithms.

- Setup $\left(1^{\lambda}, \mathrm{id}\right)$ is the remote program generation algorithm, which is run on the local machine to initialise a fresh instance of the AttKE protocol under party identifier id. On input the security parameter and id, it will output the code for a program RemKE and the initial state $\mathrm{st}_{L}$ of the LOCKE $_{K}$ algorithm. This algorithm is run locally.

- Rem $\mathrm{KE}$ (which is generated dynamically by Setup) is a program that will be run as a part of an IEE process in the remote machine, and it will keep the entire remote state of the key exchange protocol in that protected environment.

- $\operatorname{Loc}_{K E}\left(\mathrm{st}_{L}, \mathrm{~m}\right)$ is the algorithm that runs the local end of the AttKE protocol, interacting with Rem $\mathrm{KE}$. On input its current state and an incoming message $m$, it will output an updated state and an outgoing message. 
When analysing the security of such a protocol we will impose that the LocKE algorithm and all RemKE programs that may be produced by Setup keep in their state the same information that was imposed on general key exchange algorithms in Section 3. We will refer to the instances of local key exchange executions as $\operatorname{Loc}_{\mathrm{KE}}^{s}$, for $s \in \mathbb{N}$, as we will concentrate on the simplified scenario of a single local id. We will enumerate over remote instances as Rem $\mathrm{KE}_{\mathrm{i}}^{i, j}$ for $i, j \in \mathbb{N}$, and observe that the value of variable oid in this case will be set during the execution of the program itself. An AttKE is correct if, after a complete (honest) run between two participants, one local and one remote, and where the remote program is always the one to initiate the communication, both reach the accept state, both derive the same key and session identifier and have matching partner identifier strings.

ExECUTION ENVIRONMENT. The specific flavour of key exchange that we will be considering is clarified by the execution environment in Figure 6. This follows the standard modelling of active attackers, e.g. [21], when one excludes the possibility of corruption (which we do only for the sake of simplicity). There are, however, two modifications that attend to the fact that AttKE remote programs are designed to be executed under attested computation guarantees. On one hand, the adversary is given the power to create as many remote AttKE programs as it may need, by using the NewLocal oracle, revealing the entire code of the remote AttKE program to the adversary. This captures the fact that remote AttKE programs will be loaded into IEE execution environments in an otherwise untrusted remote machine, and it implies that remote AttKE programs cannot keep any long term secret information. Intuitively, this limitation will be compensated by the attested computation protocol. On the other hand, the adversary is able to freely interact with remote processes, but it is constrained in its interaction with the local machine. Indeed, the SendLocal oracle filters which messages the adversary can deliver to the local machine by checking that these are consistent with at least one remote process that the adversary is interacting with. This captures the fact that AttKE is designed to interact over a partially authenticated channel from the remote machine to the local machine, which will be provided by an attested computation protocol.

PARTNERING. We will consider the natural extension of the partnering properties introduced for passive key exchange in Section 3 to the AttKE setting. Due to space constraints, we present only the modifications to the definitions and give the details in the full version[2]. In addition to the syntactic modifications that result from referring to $\operatorname{Loc}_{\mathrm{KE}}^{s}$ and $\operatorname{Rem}_{\mathrm{KE}}^{i, j}$, we further restrict validity so that partnering is only valid when it occurs between local and remote instances, in which the latter is the initiator. As before, we will consider that an adversary violates entity authentication if he can get a session to accept, but there is no unique and confirmed valid session in its intended partner.

SECURITY. Again, the set of TestLoc and TestRem queries must be restricted in order to exclude trivial attacks. An

\begin{tabular}{|c|c|}
\hline $\begin{array}{l}\frac{\text { Game } \operatorname{Att}_{\text {AttKE, } \mathcal{A}}\left(1^{\lambda}\right):}{\text { InsList } \leftarrow[] ; \text { fake } \leftarrow}[] \\
i \leftarrow 0 \\
b \leftarrow \$\{0,1\} \\
b^{\prime} \leftarrow \$ \mathcal{A}^{\mathcal{O}}\left(1^{\lambda}, \text { id }\right) \\
\text { Return } b=b^{\prime}\end{array}$ & 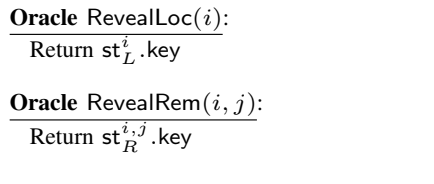 \\
\hline $\begin{array}{l}\text { Oracle } \operatorname{NewLoc}(): \\
i \leftarrow i+1 ; T_{L}^{i} \leftarrow[] \\
\left(\operatorname{Rem}_{\mathrm{KE}}^{i}, \mathrm{st}_{L}^{i}\right) \leftarrow \$ \operatorname{Setup}\left(1^{\lambda}, \mathrm{id}\right) \\
\text { InsList }[i] \leftarrow 0 \\
\text { Return } \operatorname{Rem}_{\mathrm{KE}}^{i}\end{array}$ & $\begin{array}{l}\text { Oracle } \operatorname{NewRem}(i): \\
\text { InsList }[i] \leftarrow \operatorname{InsList}[i]+1 \\
j \leftarrow \operatorname{lnsList}[i] \\
T_{R}^{i, j} \leftarrow[] ; \text { st }_{R}^{i, j} \leftarrow \epsilon \\
\text { Return } \epsilon\end{array}$ \\
\hline $\begin{array}{l}\text { Oracle } \operatorname{TestLoc}(i): \\
\text { If } \mathrm{st}_{\mathrm{L}}^{i} \cdot \delta \neq \operatorname{accept} \text { return } \perp \\
\text { If } b=0 \text { return st }{ }_{L}^{i} \cdot \text { key } \\
\text { Return fake }\left(\mathrm{st}_{L}^{i} \cdot \text { key }\right)\end{array}$ & $\begin{array}{l}\text { Oracle TestRem }(i, j): \\
\text { If } \mathrm{st}_{\mathrm{R}}^{i, j} \cdot \delta \neq \text { accept return } \perp \\
\text { If } b=0 \text { return st }{ }_{R}^{i, j} \cdot \text { key } \\
\left.\text { Return fake( } \text { st }_{R}^{i, j} \cdot \text {.key }\right)\end{array}$ \\
\hline $\begin{array}{l}\text { Oracle SendLoc }(\mathrm{m}, i): \\
\text { If } \nexists j,\left(\mathrm{~m}: T_{L}^{i}\right) \sqsubseteq T_{R}^{i, j} \text { return } \perp \\
\left(\mathrm{m}^{\prime}, \mathrm{st}_{L}^{i}\right) \leftarrow \$ \operatorname{Loc}_{\mathrm{KE}}^{i}\left(\mathrm{st}_{L}^{i}, \mathrm{~m}\right) \\
T_{L}^{i} \leftarrow \mathrm{m}^{\prime}: \mathrm{m}: T_{L}^{i} \\
\text { If } \mathrm{st}_{L}^{i} \cdot \delta \in\{\text { accept, derived }\}: \\
\quad \text { If }\left(\mathrm{st}_{L}^{i} \cdot \text { key, key*) } \notin \text { fake: }\right. \\
\quad \text { key }^{*} \leftarrow \$\{0,1\}^{\lambda} \\
\quad \text { fake } \leftarrow\left(\mathrm{st}_{L}^{i} \cdot \text { key, key }{ }^{*}\right): \text { fake } \\
\text { Return }\left(\mathrm{m}^{\prime}, \mathrm{st}_{L}^{i} \cdot \mathrm{sid}, \mathrm{st}_{L}^{i} \cdot \delta, \mathrm{st}_{L}^{i} \cdot \mathrm{pid}\right)\end{array}$ & $\begin{array}{l}\text { Oracle SendRem }(\mathrm{m}, i, j): \\
/ / \text { No restriction } \\
\mathrm{m}^{\prime} \leftarrow \$ \operatorname{Rem}_{\mathrm{KE}}\left[\mathrm{st}_{R}^{i, j}\right](\mathrm{m}) \\
T_{R}^{i, j} \leftarrow \mathrm{m}^{\prime}: \mathrm{m}: T_{R}^{i, j} \\
\text { If } \mathrm{st}_{R}^{i, j} \cdot \delta \in\{\text { accept, derived }\}: \\
\quad \text { If }\left(\mathrm{st}_{R}^{i, j} \cdot \text { key, key }{ }^{*}\right) \notin \text { fake: } \\
\quad \text { key }^{*} \leftarrow \$\{0,1\}^{\lambda} \\
\quad \text { fake } \leftarrow\left(\mathrm{st}_{R}^{i, j} \cdot \text { key, key* }\right): \text { fake }^{i, j} \\
\text { Return }\left(\mathrm{m}^{\prime}, \mathrm{st}_{R}^{i, j} \cdot \mathrm{sid}, \mathrm{st}_{R}^{i, j} \cdot \delta, \mathrm{st}_{R}^{i, j} \cdot \mathrm{pid}\right)\end{array}$ \\
\hline
\end{tabular}

Figure 6: Execution environment for AttKEs.

adversary is legitimate if it respects the following freshness criteria:

- For all TestLoc $(i)$ queries, the following holds:

1. RevealLoc $(i)$ was not queried; and

2. for all $\operatorname{Rem}_{\mathrm{KE}}^{j, k}$ s.t. $\mathrm{P}\left(\operatorname{Rem}_{\mathrm{KE}}^{j, k}, \operatorname{Loc}_{\mathrm{KE}}^{s}\right)=\mathrm{T}$, RevealRem $(j, k)$ was not queried.

- For all TestRem $(i, j)$ queries, the following holds:

1. RevealRem $(i, j)$ was not queried; and

2. for all $\operatorname{Loc}_{\mathrm{KE}}^{k}$ s.t. $\mathrm{P}\left(\operatorname{Loc}_{\mathrm{KE}}^{k}, \operatorname{Rem}_{\mathrm{KE}}^{i, j}\right)=\mathrm{T}$, RevealLoc $(i)$ was not queried.

We only consider legitimate adversaries, and say that the winning event guess occurs if $b=b^{\prime}$ at the end of the experiment. We define AttKE security by requiring both mutual authentication of parties and and key secrecy.

Definition 9 (AttKE security). An AttKE protocol is secure if, for any ppt adversary in Figure 6, and for any local party identifier string id: 1. the adversary violates entity authentication with negligible probability; and 2. its key secrecy advantage $2 \cdot \operatorname{Pr}[$ guess ] -1 is negligible.

Generic Construction. We now present a construction of an AttKE scheme from any passively secure key exchange protocol, relying additionally on a existentially unforgeable signature scheme. The intuition here is that the attested computation protocol guarantees correct remote execution of a program, but does not ensure uniqueness, i.e., it does not exclude that potentially many replicas of the same key exchange protocol instance could be running in the remote machine. By binding a fresh signature verification key with the identifier for the remote party associated with the key exchange protocol and generating a fresh nonce at the start of every execution, we can remotely execute the key exchange code whilst ensuring one-to-one authentication at the process level. This transformation can be seen as a weaker 


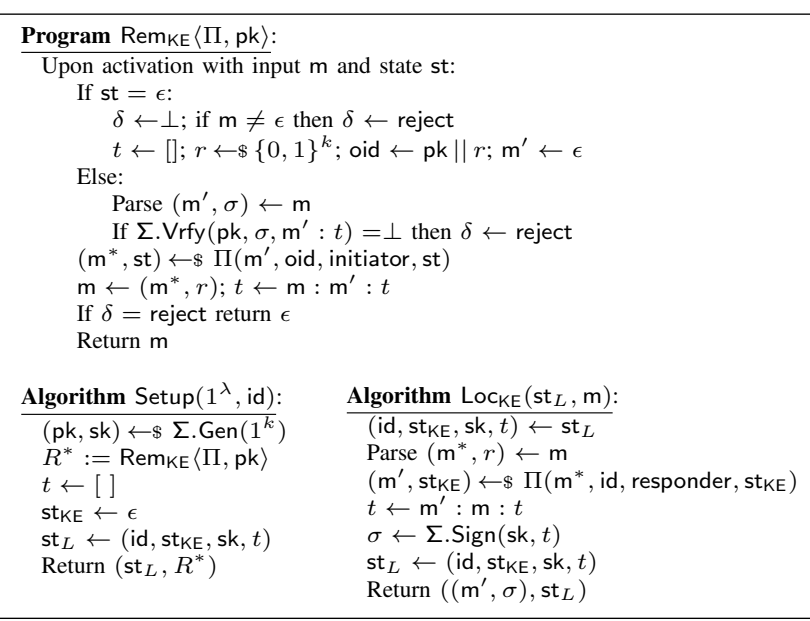

Figure 7: Details of the AttKE construction.

version of the well-known passive-to-active compilation process by Katz et al. [21], since our target security model is not fully active. We now present the details.

Consider a passively-secure authenticated key exchange protocol $\Pi$ and a signature scheme $\Sigma=$ (Gen, Sign, Vrfy). Our construction splits the execution of $\Pi$ between the local machine and a remote isolated execution environment: the responder will run locally and the initiator will run remotely within a program Rem $_{\mathrm{KE}}{ }^{3}$ The code of the remote program will have hardwired into it a unique verification key for the signature scheme. The first activation of Rem $K E$ initialises an internal state and computes a nonce, together with the first message in the key exchange protocol. The party identifier string of the remote process will then be defined to comprise the verification key and the nonce. The local part of the protocol signs the full communication trace so far. Subsequent activations of remote program Rem $\mathrm{KE}_{\mathrm{K}}$ will simply respond according to the key exchange protocol description, rejecting all inputs that fail signature verification. The details of our construction are shown in Figure 7.

- Setup first generates a fresh key pair for the signature scheme and constructs program Rem $\mathrm{KE}$, parametrised by algorithm $\Pi$ and verification key $\mathrm{pk}$, as described in Figure 7 (top). In this program state variables $\delta, \rho$, key, sid and pid are all shared with $\Pi$ (this is implicit in the figure). The initial value of $\mathrm{st}_{L}$ will store id, along with the initially empty state for the key exchange st $_{K} E$, the signing key for the signature scheme and an initially empty trace $t \log$.

- Loc KE takes $\left(\mathrm{st}_{L}, \mathrm{~m}\right)$ runs $\Pi\left(\mathrm{m}\right.$, id, responder, $\left.\mathrm{st}_{\mathrm{KE}}\right)$ to compute the next message $o$, produces signature $\sigma$ of the entire updated protocol trace, and returns the updated state $\mathrm{st}_{L}$ and message $(o, \sigma)$.

The following theorem establishes the correctness and security of the generic construction.

3. Setting the remote machine as the initiator of the protocol is the most common scenario. We considered it for simplicity; the converse can be treated analogously.
Theorem 2. Given a correct passively secure key exchange protocol $\Pi$ and an existentially unforgeable signature scheme $\Sigma$, the generic construction above yields a correct and secure AttKE protocol.

The full proof is given in the full version [2].The intuition behind the proof is that each local instance of the key exchange protocol is bound to a verification key for the signature scheme which is hardwired into the party identifier of the associated remote program code. Each remote instance of the code initialises its own party identifier by attaching a nonce to the verification key. This nonce is also transmitted along with every remote-to-local message. These facts combined with the restriction on the SendLoc oracle imply that the adversary is essentially restricted to function in a passive way, by passing around messages between the two Send oracles. Therefore, the security guarantees can be reduced to the security of the underlying key exchange protocol.

Utility. As an intermediate result building up to the construction of full-fledged authenticated and private remote attested computation, we will now present a utility theorem that describes precisely the guarantees one obtains when combining an attested computation protocol with an AttKE. Intuitively, this theorem states that attested computation guarantees that the authentication and secrecy assurance offered by AttKE are retained when we use it to establish session keys with remote IEEs, in the presence of fully active adversaries that control the remote machine, and when the key exchange is composed with arbitrary programs.

Figure 8 shows an idealised game where an adversary must distinguish between two remote machines where an AttKE scheme is executed in combination with an AC scheme. Machine $\mathcal{M}_{R}$ is any standard remote machine that is supported by the attested computation protocol, whereas $\mathcal{M}_{R}^{\prime}$ represents a modification of $\mathcal{M}_{R}$ where one can tweak the operation of Rem $\mathrm{KE}$ programs. The differences of $\mathcal{M}_{R}^{\prime}$ with respect to $\mathcal{M}_{R}$ are concentrated on the Run interface, which now operates as follows:

- It takes as additional parameters a list fake of pairs of keys and Boolean flag tweak that, when activated, identifies a process that is running an instance of Rem $\mathrm{KE}_{\mathrm{E}}$ composed with some program $Q$. This flag triggers the following modifications with respect to the operations of $\mathcal{M}_{R}$.

- When it detects that RemKE has transitioned into derived or accept state, it will check if the derived key exists in list fake. If not, it generates a new random key*, and (key, key*) is added to the list.

- When it detects that program $Q$ is set to start executing, rather than using the key as an input to $\phi$, it uses fake(key) instead.

The environment presented to the adversary models a standard attested computation interaction, where it is given total control over the remote machine using oracles Load and Run (these oracles will either give access to $\mathcal{M}_{R}$ or to $\mathcal{M}_{R}^{\prime}$, depending on a secret bit $b$ generated in the beginning of the game). The adversary is also able to obtain 
challenge remote programs using a $\operatorname{NewSession}(Q)$ oracle that uses the attested computation scheme to compile Rem $\mathrm{KE}$ composed with arbitrary program $Q$ of its choice under a mapping function $\phi_{\text {key }}$ that reveals the relevant parts of the key exchange state (namely the secret key key, the party identifiers oid and pid, the state $\delta$ and the session identifier sid). We observe that such arbitrary programs can leak all of the information revealed by $\phi_{\text {key }}$ to the attacker. If the adversary chooses to Load a challenge program, and if $\mathcal{M}_{R}^{\prime}$ is being used in the game, then it will be tweaked as described above. Whenever $\operatorname{NewSession}(Q)$ is called, the environment creates a new local session $i$ that the adversary can interact with using a Send $(i, \mathrm{~m})$ oracle. The Send oracle uses the Verify algorithm of the attested computation scheme to validate attested outputs and, if they are accepted, feeds them to the Loc KE instance (and also ensures that list fake is updated). Finally, the adversary can explicitly choose to be tested (as opposed to the implicit testing it may trigger using arbitrary programs $Q$ ) by calling Test on a local instance. This oracle will either return the true key, if $b=0$, or the associated random key that is kept in the fake list. As before, we define the winning event guess to occur when $b=b^{\prime}$ in the end of the game.

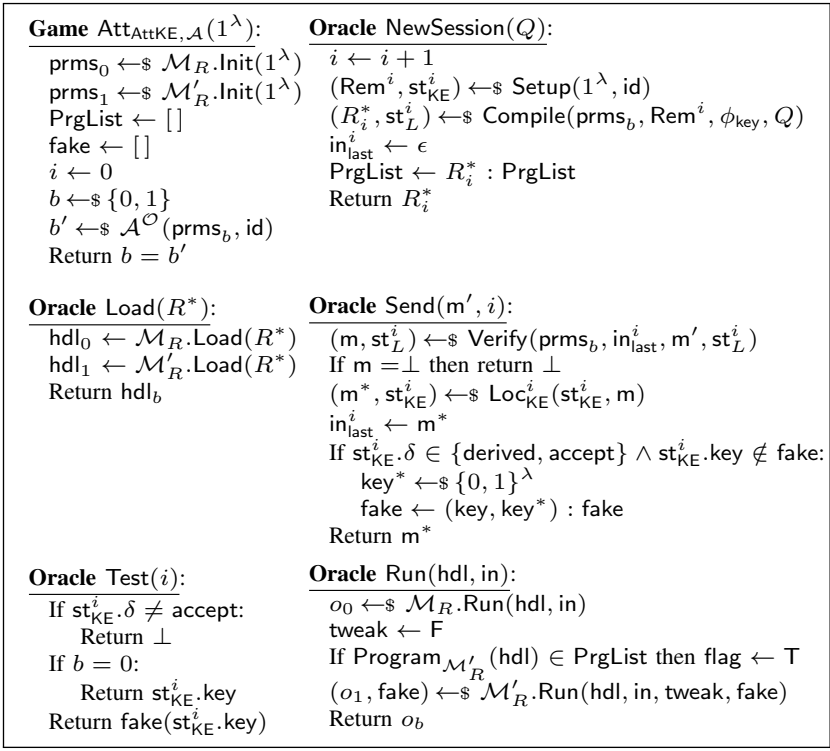

Figure 8: Game defining the utility of an AttKE scheme when used in the context of attested computation.

The proof of the following theorem can be found in the full version of the paper [2].

Theorem 3 (AttKE utility). If AttKE is correct and secure and the AC protocol is correct, secure and ensures minimum leakage, then for all ppt adversaries in the utility experiment: 1 . the probability that the adversary violates AttKE two-sided entity authentication is negligible; and the key secrecy advantage $2 \cdot \operatorname{Pr}[$ guess ] -1 is negligible.

The intuition behind the proof is that one can use the security of attested computation to exclude Send queries that do not match a legitimate remote trace. This essentially maps to the restriction in the AttKE security game imposed on the SendLoc oracle. The proof is concluded by applying the minimum leakage property of the attested computation protocol to show that any attack by the adversary against AttKE when it is run inside a remote machine can be transformed (via trace simulation) to an attack against the original AttKE when it is run in source code form.

\section{Secure Outsourced Computation}

In this section we build on the results in previous sections to design and analyze a protocol for secure outsourced computation. Informally, we require two properties: i) that only the legitimate local user can pass inputs to the outsourced program and ii) that the $\mathrm{I} / \mathrm{O}$ of the remote program is secret from any observer (even an actively malicious one).

We first give syntax for the protocols that solve this problem, then propose formal definitions for the properties that we outlined above, and conclude with a generic construction that combines a key-exchange for attestation, a scheme for attested computation and an authenticated encryption scheme.

SyNTAX. A Secure Outsourced Computation scheme (SOC) for a remote machine $\mathcal{M}_{R}$ is defined by the following algorithms:

- Compile(prms, $P$, id) is the program compilation algorithm. On input public parameters prms, a program $P$ and a party identifier id, it outputs a compiled program $P^{*}$, together with an initial state $s_{l}$ for the local side algorithms. We assume that initially $\mathrm{st}_{l}$.accept $=\perp$. Note that unlike the AC compilation algorithm, this algorithm only takes one program as input, as this scheme is intended for providing guarantees for the whole trace and not only for an initial segment.

- BootStrap (prms, $o$, st $\left._{l}\right)$ is the client side initialization algorithm. On input public parameters prms, $o$ (presumably the last message from the remote machine) and local state $\mathrm{st}_{l}$, it returns the next message $i$ to be delivered to the remote machine in the bootstrapping step step, together with the updated local state. We assume BootStrap sets an accept flag to $T$ when the initialization process successfully terminates.

- Verify $\left(\mathrm{prms}, o^{*}, \mathrm{st}_{l}\right)$ is the verification algorithm. It fulfills the same function as the AC verification algorithm. Note that, as all the inputs are provided by the local machine, we do not need to feed it the last input as it can be stored in the state. It is expected to return $\perp$ if $\mathrm{st}_{l}$.accept $\neq \mathrm{T}$.

- Encode(prms, $\left.i, \mathrm{st}_{l}\right)$ is the encoding algorithm. On input the public parameters, local state and the next intended input for $P$, it returns the next input $i^{*}$ for $P^{*}$ together with the updated local state. It is expected to return $\perp$ if st $_{l}$.accept $\neq \mathrm{T}$.

- Attest (prms, hdl, $i$ ) is, as in an AC scheme, the (untrusted) attestation algorithm.

A party $A$ with identifier id who wants to outsource program $P$ to the remote machine first compiles $P$ with his id, 
thus obtaining $P^{*}$ and some secret data st $t_{l}$. He then loads $P^{*}$ on the remote machine using some untrusted protocol. As it is, the program $P^{*}$ is not ready to receive inputs intended for $P$ : an initial bootstrapping phase (until BootStrap sets the accept flag) is necessary to establish some shared secrets between the IEE in which $P^{*}$ is executed and $A$. Then when $A$ wants to send an input to the remote execution, he encodes it using Encode, sends it (using Attest) and verifies the output provided by Attest using Verify.

In this section, for simplicity reasons, we assume that the program $P$ is deterministic. However, as for an $\mathrm{AC}$ scheme it would be easy to extend all the definitions to a non-deterministic program.

INPUT INTEGRITY. While security of attested computation aims at ensuring that a trace was honestly produced on the remote side, it does nothing to restrict the provenance of the inputs received.

We provide a stronger notion named input integrity which, intuitively, ensures that if a program is compiled by a party with identifier id, then only that party may use the remote compiled program. We ensure this property by making sure that the local and remote views coincide (up to the last message exchanged, which may not have yet been delivered). The following formula $\Psi$ which relates two input/output traces captures this intuition.

$$
\Psi\left(T, T^{\prime}\right):=T=T^{\prime} \vee \exists o .\left(T=o:: T^{\prime}\right) \exists i .\left(T^{\prime}=i:: T\right)
$$

The formalization that we provide in Figure 9 is as follows. The adversary chooses a program $P$ that is compiled with an honest party's id yielding $P^{*}$ (which is given to the adversary). The adversary is given access to two oracles. A bootstrapping oracle that simply executes BootStrap honestly; and a send oracle that verifies the last (presumed) output of the remote program and encodes the next input (which is provided by the adversary), while keeping track of the local view of the trace. The goal of the adversary is then create a mismatch between the local and remote view of the trace.

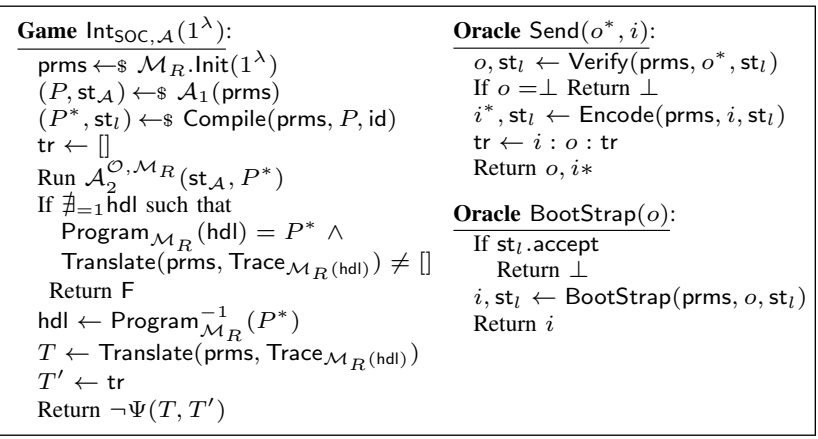

Figure 9: Input integrity of a SOC scheme

Definition 10 (Input Integrity). We say that a SOC scheme satisfies input integrity if there exists a polynomial time algorithm Translate such that for all ppt $\mathcal{A}$ the experiment described in Figure 9 returns true with probability negligible in the security parameter.
INPUT PRIVACY. We define the privacy of I/O with an indistinguishability game. One important point here is that we chose to restrict the class of programs we consider to length-uniform (written lu) programs. A program is length uniform if the length of its outputs depends only on the length of its inputs. Intuitively, this is because the encryption scheme is allowed to leak the length of the messages, which in turn would leak information about the inputs for a non lu program.

The formalization described in Figure 10 is as follows. We start by choosing a bit $b$ that will determine whether the adversary will be talking with the left send oracle or the right send oracle (described later). As for input integrity, the adversary then chooses a program $P$. We compile it for an honest party's identifier and give the resulting $P^{*}$ to the adversary. The adversary is also given access to the bootstrapping oracle. In addition, he is given access to a left or right send oracle. This oracle, on a request with the last candidate output of the remote machine and two inputs $i_{0}$ and $i_{1}$, verifies the last candidate output and, depending on the bit $b$, encodes either $i_{0}$ or $i_{1}$ and returns the result. The goal of the adversary is to guess the bit $b$ with non-negligible bias from $1 / 2$.

\begin{tabular}{|c|c|}
\hline $\begin{array}{l}\text { Game Privsoc, } \mathcal{A}\left(1^{\lambda}\right): \\
b \leftarrow \$\{0,1\} \\
\text { prms } \leftarrow \mathcal{M}_{R} \cdot \text { Init }\left(1^{\lambda}\right) \\
\left(P, \text { st }_{\mathcal{A}}\right) \leftarrow \$ \mathcal{A}_{1}(\text { prms }) \\
\text { If } \neg \operatorname{lu}(P) \operatorname{Return} b^{\prime} \leftarrow \$\{0,1\} \\
\left(P^{*}, \text { st }_{l}\right) \leftarrow \$ \text { Compile }(\text { prms }, P, \text { id }) \\
b^{\prime} \leftarrow \mathcal{A}_{2}\left(\text { st }_{\mathcal{A}}, P^{*}\right) \mathcal{O}, \mathcal{M}_{R} \\
\text { Return } b=b^{\prime}\end{array}$ & $\begin{array}{l}\text { Oracle } \operatorname{Send}_{b}\left(o^{*}, i_{0}, i_{1}\right): \\
o, \text { st }_{l} \leftarrow \text { Verify }\left(\operatorname{prms}, o^{*}, \text { st }_{l}\right) \\
\text { If }\left|m_{0}\right| \neq\left|m_{1}\right| \operatorname{Return} \perp \\
i^{*}, \text { st }_{l} \leftarrow \operatorname{Encode}\left(\operatorname{prms}, i_{b}, \text { st }_{l}\right) \\
\text { Return } i^{*} \\
\text { Oracle BootStrap }(o): \\
\text { If st } \text { st }_{l} \cdot \operatorname{accept} \\
\quad \operatorname{Return} \perp / /(1 \text { init max }) \\
i, \text { st }_{l} \leftarrow \operatorname{BootStrap}\left(\operatorname{prms}, o, \text { st }_{l}\right) \\
\text { Return } i\end{array}$ \\
\hline
\end{tabular}

Figure 10: Input privacy of a SOC scheme

Definition 11. We say that a SOC scheme satisfies input privacy if, for all ppt $\mathcal{A}$, the experiment in Figure 10 returns true with probability $1 / 2$ up to a negligible function.

This definition ensures that there exist no two traces (with messages of the same length) played by an honest party over a SOC protocol that are distinguishable for an (active) adversary. This means that no adversary can gain information on the inputs sent out by a local machine using a SOC scheme, besides the length of the messages exchanged, achieving our goal of hiding the honest party's inputs.

Definition 12. We say that a SOC scheme is secure if it satisfies both input privacy and input integrity.

AN IMPLEMENTATION OF A SECURE SOC SCHEME. Having defined what security we expect from a SOC scheme, we now define a scheme that satisfies these requirements. We base our construction on an AttKE, and an AC scheme. The main idea is using the AttKE to establish a key between the party agent and the IEE, and then communicate with the IEE over the secure channel established with this key.

Formally, let (Compile, Attest, Verify) be an AC scheme, (Setup, LocKE) be an AttKE and $(E, D, K)$ be an authenticated encryption scheme. Figure 11 defines a SOC scheme. 
The most important part is the compilation part, which uses the AC scheme compilation to compile the composition of the RemKE program generated by Setup together with program $P$ running over a secure channel (denoted by $C(P)$ ). The initial local state is the union of the state provided by the AC compilation and the AttKE setup. The program $C(P)$ simply decrypts the message it receives checks that the sequence number of the message matches its view the passes the decrypted message to $P$. It then retrieves the output of $P$, appends the corresponding next sequence number and outputs it. This mechanism ensures that all messages received (resp. sent out) by $P^{*}$ after the bootstrapping phase have the form $E(i \# m, k)$ where $i$ is the position of the message in the trace, $m$ is the message intended to (resp. produced by) $P$, and $k$ is the key established by the AttKE.

On the local side, the bootstrapping mechanism simply consists of running the local $\mathrm{KE}$ over the $\mathrm{AC}$ protocol as already described in the utility definition. Once the key has been established, the local state keeps track of the local view of the sequence number. Verifying an output consists in decrypting it and checking that the sequence number against the local view of it. Encoding an input, is just appending the correct sequence number and encrypting it with the shared key.

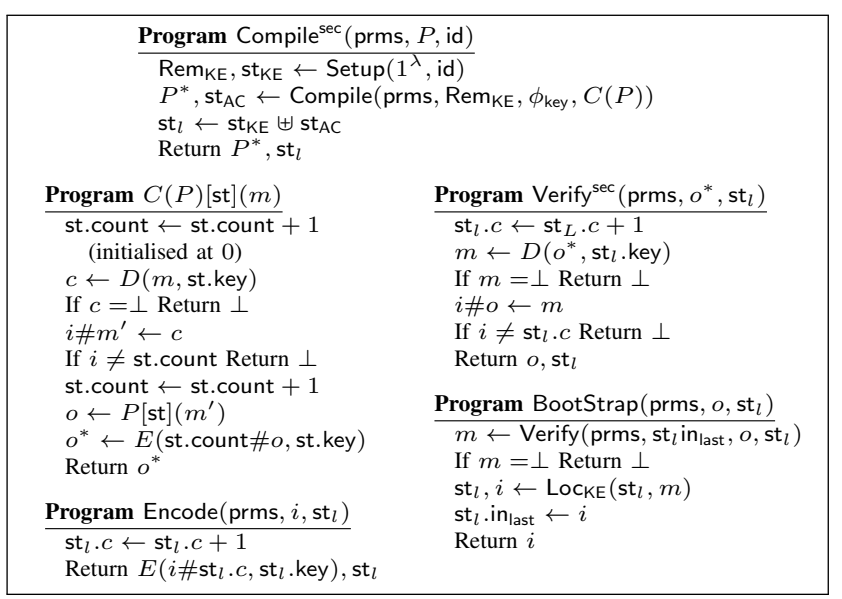

Figure 11: SOC algorithms

Theorem 4. If (Compile, Attest, Verify) is a correct and secure AC scheme, $\left\{\right.$ Setup, Loc $\left.\mathrm{KE}, \operatorname{Rem}_{\mathrm{KE}}\right\}$ is a secure At$t K E$ and $(E, D, K G)$ is an secure authenticated encryption scheme, then the SOC presented in Figure 11 is secure.

The complete proof can be found in the full version of the paper[2], we provide here a sketch of proof, following the game hopping paradigm. We first do a game hop that consists in replacing the key established by $P^{*}$ and the party using the AttKE by a "magically" shared fresh key. The utility property of the AttKE provides us with the fact that we can replace the key shared by the remote machine and the local agent by a freshly generated key. We are left with showing that this key is shared with an IEE which is indeed running $P^{*}$ and not Compile( $\left.\operatorname{Rem}_{\mathrm{KE}}, \phi_{\mathrm{key}}, Q\right)$ for some other $Q$, this is provided by the security of the $\mathrm{AC}$ scheme.

We then prove input integrity by remarking that injecting new messages in the trace would contradict the unforgeability of the authenticated encryption scheme. The sequence number ensures that the messages are delivered in the right order and that replays are impossible.

We remark that the input integrity property ensures that we know that the only meaningful action the adversary can take is to forward messages between the remote and local machines. Taking advantage of that fact we can reduce the input privacy game to the IND-CPA property of the authenticated encryption.

\section{Conclusion}

This paper offers a set of building blocks for constructing protocols that leverage the guarantees of IEEs. First, we define and construct attested computation based on IEEs. In the process we identify and formalize two key properties that such protocols need to satisfy to be useful: composition awareness and minimal leakage. Our instantiation of attested computation relies on SGX.

Next, we provide key-exchange protocols between a remote party and an IEE. These protocols are essential components for any construction where secrecy of data communicated to and from the IEE is important. Our contribution is to adapt existing models of security for key-exchange to the novel setting where protocol participants do not necessarily have an a-priori identity - IEEs cannot be uniquely identified before actively communicating with them. For constructions, we present a modular approach where by combining a passively secure key exchange protocol with an arbitrary attested computation protocol we obtain a fully secure keyexchange protocol. As an application, we show how to use attested computation, key-exchange and symmetric authenticated encryption to generically construct a secure outsourced computation scheme for arbitrary functionalities.

In terms of follow-up work, the natural next step is to use the building blocks that we provide to construct other protocols. One interesting target (which generalizes the application in this paper) is to construct secure multi-party computation based on IEEs and experimentally compare their efficiency with the existent software-only alternatives.

\section{Acknowledgements}

This work was supported by the European Union's 7th Framework Program (FP7/2007-2013) under grant agreement n. 609611 (PRACTICE). The authors would also like to thank the anonymous reviewers for their valuable comments and suggestions to improve the quality of the paper.

\section{References}

[1] I. Anati, S. Gueron, S. Johnson, and V. Scarlata. Innovative technology for cpu based attestation and sealing. In Workshop on Hardware and Architectural Support for Security and Privacy, page 10, 2013. 
[2] Manuel Barbosa, Bernardo Portela, Guillaume Scerri, and Bogdan Warinschi. Foundations of hardware-based attested computation and application to SGX. Cryptology ePrint Archive, Report 2016/014, 2016. http://eprint.iacr.org/.

[3] Andrew Baumann, Marcus Peinado, and Galen C. Hunt. Shielding applications from an untrusted cloud with haven. In 11th USENIX Symposium on Operating Systems Design and Implementation, OSDI '14, Broomfield, CO, USA, October 6-8, 2014., pages 267-283. USENIX Association, 2014.

[4] Mihir Bellare, Joe Kilian, and Phillip Rogaway. The security of cipher block chaining. In Advances in Cryptology - CRYPTO '94, 14th Annual International Cryptology Conference, Santa Barbara, California, USA, August 21-25, 1994, Proceedings, volume 839 of Lecture Notes in Computer Science, pages 341-358. Springer, 1994.

[5] Siavosh Benabbas, Rosario Gennaro, and Yevgeniy Vahlis. Verifiable delegation of computation over large datasets. In Advances in Cryptology - CRYPTO 2011 - 31st Annual Cryptology Conference, Santa Barbara, CA, USA, August 14-18, 2011. Proceedings, volume 6841 of Lecture Notes in Computer Science, pages 111-131. Springer, 2011.

[6] Ernest F. Brickell, Jan Camenisch, and Liqun Chen. Direct anonymous attestation. In Proceedings of the 11th ACM Conference on Computer and Communications Security, CCS 2004, Washington, DC, USA, October 25-29, 2004, pages 132-145. ACM, 2004.

[7] Ernie Brickell, Liqun Chen, and Jiangtao Li. A new direct anonymous attestation scheme from bilinear maps. In Trusted Computing - Challenges and Applications, First International Conference on Trusted Computing and Trust in Information Technologies, Trust 2008, Villach, Austria, March 11-12, 2008, Proceedings, volume 4968 of Lecture Notes in Computer Science, pages 166-178. Springer, 2008.

[8] Christina Brzuska, Nigel P. Smart, Bogdan Warinschi, and Gaven J. Watson. An analysis of the EMV channel establishment protocol. In 2013 ACM SIGSAC Conference on Computer and Communications Security, CCS'13, Berlin, Germany, November 4-8, 2013, pages 373386. ACM, 2013.

[9] Ran Canetti. Universally composable security: A new paradigm for cryptographic protocols. In 42nd Annual Symposium on Foundations of Computer Science, FOCS 2001, 14-17 October 2001, Las Vegas, Nevada, USA, pages 136-145. IEEE Computer Society, 2001

[10] Luigi Catuogno, Alexandra Dmitrienko, Konrad Eriksson, Dirk Kuhlmann, Gianluca Ramunno, Ahmad-Reza Sadeghi, Steffen Schulz, Matthias Schunter, Marcel Winandy, and Jing Zhan. Trusted virtual domains - design, implementation and lessons learned. In Trusted Systems, First International Conference, INTRUST 2009, Beijing, China, December 17-19, 2009. Revised Selected Papers, volume 6163 of Lecture Notes in Computer Science, pages 156-179. Springer, 2009.

[11] Anupam Datta, Jason Franklin, Deepak Garg, and Dilsun Kirli Kaynar. A logic of secure systems and its application to trusted computing. In 30th IEEE Symposium on Security and Privacy, SP 2009, 17-20 May 2009, Oakland, California, USA, pages 221-236. IEEE Computer Society, 2009.

[12] Aurélien Francillon, Quan Nguyen, Kasper Bonne Rasmussen, and Gene Tsudik. A minimalist approach to remote attestation. In Design, Automation \& Test in Europe Conference \& Exhibition, DATE 2014, Dresden, Germany, March 24-28, 2014, pages 1-6. European Design and Automation Association, 2014.

[13] He Ge and Stephen R. Tate. A direct anonymous attestation scheme for embedded devices. In Public Key Cryptography - PKC 2007, 10th International Conference on Practice and Theory in PublicKey Cryptography, Beijing, China, April 16-20, 2007, Proceedings, volume 4450 of Lecture Notes in Computer Science, pages 16-30. Springer, 2007.

[14] C. Gebhardt and A. Tomlinson. Secure virtual disk images for grid computing. In Third Asia-Pacific Trusted Infrastructure Technologies Conference, APTC '08, pages 19-29, Washington, DC, USA, 2008. IEEE Computer Society.
[15] Rosario Gennaro, Craig Gentry, and Bryan Parno. Non-interactive verifiable computing: Outsourcing computation to untrusted workers. In Advances in Cryptology - CRYPTO 2010, 30th Annual Cryptology Conference, Santa Barbara, CA, USA, August 15-19, 2010. Proceedings, volume 6223 of Lecture Notes in Computer Science, pages 465482. Springer, 2010.

[16] Rosario Gennaro, Craig Gentry, Bryan Parno, and Mariana Raykova. Quadratic span programs and succinct nizks without pcps. In $A d$ vances in Cryptology - EUROCRYPT 2013, 32nd Annual International Conference on the Theory and Applications of Cryptographic Techniques, Athens, Greece, May 26-30, 2013. Proceedings, volume 7881 of Lecture Notes in Computer Science, pages 626-645. Springer, 2013.

[17] Kenneth A. Goldman, Ronald Perez, and Reiner Sailer. Linking remote attestation to secure tunnel endpoints. In Proceedings of the 1st ACM Workshop on Scalable Trusted Computing, STC 2006, Alexandria, VA, USA, November 3, 2006, pages 21-24. ACM, 2006.

[18] Shafi Goldwasser, Silvio Micali, and Ronald L. Rivest. A digital signature scheme secure against adaptive chosen-message attacks. SIAM J. Comput., 17(2):281-308, 1988.

[19] Matthew Hoekstra, Reshma Lal, Pradeep Pappachan, Vinay Phegade, and Juan del Cuvillo. Using innovative instructions to create trustworthy software solutions. In HASP 2013, The Second Workshop on Hardware and Architectural Support for Security and Privacy, TelAviv, Israel, June 23-24, 2013, page 11. ACM, 2013.

[20] Intel. Software Guard Extensions Programming Reference, 2014. https://software.intel.com/sites/default/files/managed/48/88/ 329298-002.pdf.

[21] Jonathan Katz and Moti Yung. Scalable protocols for authenticated group key exchange. In Advances in Cryptology - CRYPTO 2003, 23rd Annual International Cryptology Conference, Santa Barbara, California, USA, August 17-21, 2003, Proceedings, volume 2729 of Lecture Notes in Computer Science, pages 110-125. Springer, 2003.

[22] Patrick Koeberl, Steffen Schulz, Ahmad-Reza Sadeghi, and Vijay Varadharajan. Trustlite: a security architecture for tiny embedded devices. In Ninth Eurosys Conference 2014, EuroSys 2014, Amsterdam, The Netherlands, April 13-16, 2014, pages 10:1-10:14. ACM, 2014.

[23] J. M. McCune, B. J. Parno, A. Perrig, M. Reiter, and H. Isozaki. Flicker: An execution infrastructure for tcb minimization. SIGOPS Oper. Syst. Rev., 42(4):315-328, April 2008.

[24] Microsoft. BitLocker Drive Encryption: Data Encryption Toolkit for Mobile PCs: Security Analysis, 2007. https://technet.microsoft.com/ en-us/library/cc162804.aspx.

[25] Job Noorman, Pieter Agten, Wilfried Daniels, Raoul Strackx, Anthony Van Herrewege, Christophe Huygens, Bart Preneel, Ingrid Verbauwhede, and Frank Piessens. Sancus: Low-cost trustworthy extensible networked devices with a zero-software trusted computing base. In Proceedings of the 22th USENIX Security Symposium, Washington, DC, USA, August 14-16, 2013, pages 479-494. USENIX Association, 2013.

[26] Bryan Parno, Jon Howell, Craig Gentry, and Mariana Raykova. Pinocchio: Nearly practical verifiable computation. In 2013 IEEE Symposium on Security and Privacy, SP 2013, Berkeley, CA, USA, May 19-22, 2013, pages 238-252. IEEE Computer Society, 2013.

[27] Felix Schuster, Manuel Costa, Cédric Fournet, Christos Gkantsidis, Marcus Peinado, Gloria Mainar-Ruiz, and Mark Russinovich. VC3: trustworthy data analytics in the cloud using SGX. In 2015 IEEE Symposium on Security and Privacy, SP 2015, San Jose, CA, USA May 17-21, 2015, pages 38-54. IEEE Computer Society, 2015.

[28] Sean W. Smith. Outbound authentication for programmable secure coprocessors. Int. J. Inf. Sec., 3(1):28-41, 2004.

[29] Ben Smyth, Mark Ryan, and Liqun Chen. Direct anonymous attestation (DAA): ensuring privacy with corrupt administrators. In Security and Privacy in Ad-hoc and Sensor Networks, 4th European Workshop, ESAS 2007, Cambridge, UK, July 2-3, 2007, Proceedings, volume 4572 of Lecture Notes in Computer Science, pages 218-231. Springer, 2007. 